\title{
Green Route for Fabrication of Water-Treatable Thermoelectric Generators
}

\author{
Shinichi Hata $\left(\mathbb{D},{ }^{1}\right.$ Misaki Shiraishi, ${ }^{1}$ Soichiro Yasuda, ${ }^{1}$ Gergely Juhasz, ${ }^{2}$ Yukou Du $\left(\mathbb{D},{ }^{3}\right.$ \\ Yukihide Shiraishi $\left(1,{ }^{1}\right.$ and Naoki Toshima $\oplus^{4}$ \\ ${ }^{1}$ Department of Applied Chemistry, Faculty of Engineering, Sanyo-Onoda City University, Daigaku-dori, 1-1-1, Sanyo-Onoda, \\ Yamaguchi 756-0884, Japan \\ ${ }^{2}$ Department of Chemistry, Graduate School of Science and Technology, Tokyo Institute of Technology, 2-12-1 Ookayama, Meguro- \\ ku, Tokyo 152-8551, Japan \\ ${ }^{3}$ College of Chemistry, Chemical Engineering and Materials Science, Soochow University, 199 Renai Road, Suzhou 215123, China \\ ${ }^{4}$ Tokyo University of Science, Yamaguchi, Japan
}

Correspondence should be addressed to Shinichi Hata; hata@rs.socu.ac.jp and Yukihide Shiraishi; shiraishi@rs.socu.ac.jp

Received 6 August 2021; Accepted 16 November 2021; Published 7 January 2022

Copyright (c) 2022 Shinichi Hata et al. Exclusive Licensee Beijing Institute of Technology Press. Distributed under a Creative Commons Attribution License (CC BY 4.0).

\begin{abstract}
Since future energy harvesting technologies require stable supply and high-efficiency energy conversion, there is an increasing demand for high-performance organic thermoelectric generators (TEGs) based on waterproof thermoelectric materials. The poor stability of n-type organic semiconductors in air and water has proved a roadblock in the development of reliable thermoelectric power generators. We developed a simple green route for preparing $\mathrm{n}$-type carbon nanotubes (CNTs) by doping with cationic surfactants and fabricated films of the doped CNTs using only aqueous media. The thermoelectric properties of the CNT films were investigated in detail. The nanotubes doped using a cationic surfactant (cetyltrimethylammonium chloride (CTAC)) retained an $\mathrm{n}$-doped state for at least 28 days when exposed to water and air, indicating higher stability than that for contemporary CNT-based thermoelectric materials. The wrapping of the surfactant molecules around the CNTs is responsible for blocking oxygen and water from attacking the CNT walls, thus, extending the lifetime of the n-doped state of the CNTs. We also fabricated thermoelectric power conversion modules comprising CTAC-doped (n-type) and sodium dodecylbenzenesulfonate- (SDBS-) doped (p-type) CNTs and tested their stabilities in water. The modules retained $80 \pm 2.4 \%$ of their initial maximum output power (at a temperature difference of $75^{\circ} \mathrm{C}$ ) after being submerged in water for 30 days, even without any sealing fills to prevent device degradation. The remarkable stability of our CNT-based modules can enable the development of reliable soft electronics for underwater applications.
\end{abstract}

\section{Introduction}

All energy is ultimately dissipated as heat; therefore, the management of waste heat is critical for meeting the world's increasing energy demand in an environmentally sustainable manner [1-3]. In particular, significant amounts of heat are dissipated to the environment from sources, such as automotive engine exhausts and industrial hot water pipes. Considerable attention is being devoted toward developing strategies for recovering heat from such sources $[4,5]$. Organic thermoelectric materials have shown promise for the generation of electricity from low-grade waste heat (i.e., waste heat from sources with a temperature below $150^{\circ} \mathrm{C}$ ) and are expected to play an important role in powering condition monitoring sensors around engines, motors, and so on [6-12]. Wearable sensors enable the noninvasive monitoring of physiological parameters, such as blood pressure [13] and body temperature [14], which may help in the early detection of diseases [15] and life-threatening conditions such as cerebral infarction. It is envisaged that such devices will become widespread in the future. There have been many advances in making wearable sensors energy autonomous by integrating them with thermoelectric materials that harvest energy from the environment [16]. Thermoelectric modules must satisfy three requirements to be suitable for integration in wearable devices: (1) high atmospheric, moisture, and 
thermal stability; (2) high energy conversion efficiency; and (3) mechanical flexibility. However, most conventional thermoelectric materials [16] and sensor devices reported to date [17] fail to meet all three requirements. In particular, the lack of water resistance and stability $[18,19]$ has proven to be a major impediment to developing practical wearable devices [20]. Conversely, water-resistant thermoelectric devices, which can be used in wet or underwater environments, have considerable potential for powering wearable electronics.

Carbon nanotube- (CNT-) based thermoelectric materials, which have been the focus of considerable research attention in recent years, are composed of carbon and therefore less toxic than bulk inorganic thermoelectric materials such as bismuth and tellurium [21-23]. In addition, owing to the flexibility of CNTs, it is relatively easier to fabricate CNT films than those of inorganic thermoelectric materials like bismuth and tellurium [24]. The thermoelectric performance of such a film or sheet is expressed by a dimensionless figure of merit, ZT (Equation (1)) [25], or the power factor, PF (Equation (2)).

$$
\begin{aligned}
& Z T=\frac{S^{2} \sigma}{\kappa T}, \\
& \mathrm{PF}=S^{2} \sigma,
\end{aligned}
$$

where $T$ is the absolute temperature, $S$ is the Seebeck coefficient, $\sigma$ is the electrical conductivity, and $\kappa$ is the in-plane thermal conductivity of the film. Owing to the strong correlation between $S, \sigma$, and $\kappa$, improving the PF of a thermoelectric material without sacrificing $Z T$ is difficult $[23,25]$. CNTs act as p-type conductors in the presence of water or oxygen, and the assembly of both $\mathrm{p}$ - and n-type materials is essential for efficient thermoelectric conversion [26]. For this reason, many efforts have been made in recent years to develop n-type CNTs by chemical doping with electron donors such as polyethyleneimine (PEI) $[27,28]$ or cationic salts [29] such as crown ether metal complexes [30]. Although considerable progress has been made in the synthesis of n-type CNTs, the optimal dopant/nanotube interface design of n-type CNT films and the degradation mechanism by molecular oxygen and water are still not fully understood. However, since the dopants are only soluble in nonaqueous solvents, only organic solvents can be used for the preparation of functional CNT films, making the route from fabrication to application more demanding [31]. In addition, the film fabrication procedures are not environment-friendly. The use of environment-friendly or "green" processes for CNT films is desirable. To this end, the use of water as a solvent for fabricating CNT films is preferable to using organic solvents. Nevertheless, as Nonoguchi et al. [30] have shown the solvent used for CNT film preparation has a significant effect on the thermoelectric properties of the film. This phenomenon needs to be studied in detail, because it may have important implications for the fabrication of doped CNT films using aqueous media.
To prevent the doping level and Seebeck coefficient of CNTs from reverting to those in the air-exposed state, surfactant molecules are promising doping agents, because their chemisorption on the nanotube surface can prevent the electrophilic reaction of water on the electronically activated sites. Herein, we propose a facile method for the fabrication of flexible n-type CNT films using water as the only solvent. The n-type behavior is induced by doping the CNTs with cationic surfactants. Previous reports on surfactant-doped CNTs have not elucidated the role of surfactant ionicity and alkyl chain length, despite the need for further improvement in the thermoelectric properties of the CNT-based materials [29, 32-34]. Therefore, we investigated the effect of the surfactant ionicity and alkyl chain length on the organic thermoelectric properties of the CNT films. Furthermore, to determine the exact role of the dopant, a systematic study was conducted on the effect of the preparation solvent (e.g., nonpolar solvents, polar aprotic solvents, and polar protic solvents) on the carrier conduction of CNTs. We investigated the nature of the adsorption of, and interaction between, the surfactant molecules and the CNTs to acquire an understanding of their thermoelectric properties and assessed whether the CNT films would be stable in air and underwater. Finally, we fabricated a module comprising 5 $\mathrm{p}-\mathrm{n}$ units using the surfactant-doped CNT films and studied its power generation performance and stability underwater.

\section{Materials and Methods}

2.1. Fabrication of Surfactant-Doped CNT Film in Aqueous Medium. The details of the chemicals used in this study are provided in the Supplementary Material. We prepared films from single-walled CNTs (diameter $=1.5 \pm 0.8 \mathrm{~nm}$; metallic/semiconducting ratio $=1: 2$ ) doped with three quaternary alkyl ammonium chloride surfactants $\left(\mathrm{CH}_{3}\left(\mathrm{CH}_{2}\right)_{n} \mathrm{~N}^{+}\left(\mathrm{CH}_{3}\right)_{3}\left(\mathrm{Cl}^{-}\right)\right)$: octyltrimethylammonium chloride (OTAC; $n=7)$, dodecyltrimethylammonium chloride (DTAC; $n=11$ ), and cetyltrimethylammonium chloride (CTAC; $n=15$ ). Figure 1 schematically depicts the fabrication procedure of the surfactant-doped CNT films. Briefly, $60 \mathrm{mg}$ of CNTs was dispersed in $30 \mathrm{~mL}$ of an $11 \mathrm{mM}$ aqueous solution of the surfactant in a $100 \mathrm{~mL}$ tall-form beaker by a $15 \mathrm{~min}$ ice-bath ultrasonic treatment (Ultrasonic Cleaner, TAITEC; Branson Sonifier 250D, Central Scientific, Tokyo, Japan). The resulting CNT suspension was immediately filtered using a polytetrafluoroethylene (PTFE) membrane filter (pore size: $1.0 \mu \mathrm{m}$ ). The film deposited on the filter was rinsed with $4 \mathrm{~L}$ of water to remove excess surfactant and then allowed to stand for $3 \mathrm{~h}$. The films were peeled from the filter membrane and dried overnight in a vacuum oven $\left(60^{\circ} \mathrm{C}\right.$, $<0.1 \mathrm{MPa})$. The dry, freestanding doped CNT films (Figure S1) had a thickness of $20 \pm 4.0 \mu \mathrm{m}$. The sample was cut into film sizes for thermoelectric measurements. Other surfactant-doped CNT films were also prepared, taking into account the appropriate concentrations of reagents. The procedures for the fabrication of CNT films using various organic solvents, characterization of the films, and evaluation of the flexible thermoelectric generator (TEG) are described in the Supplementary Material. 


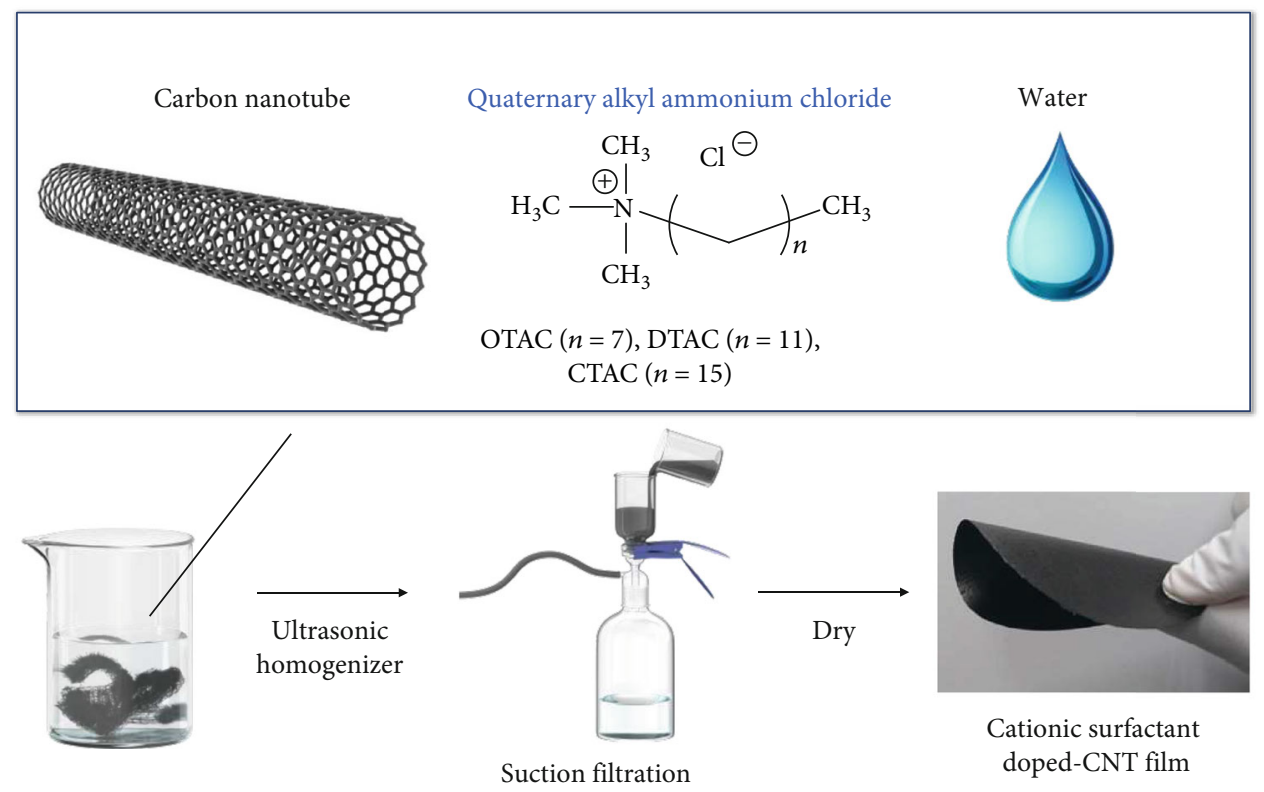

FIGURE 1: Schematic depiction of the procedure for the fabrication of cationic surfactant-doped CNT films. Ultrasonic homogenization was carried out for $15 \mathrm{~min}$ in an ice bath. Filtration was carried out using a PTFE membrane filter ( $1 \mu \mathrm{m}$ pore size). Before drying, the deposited film was washed with $4 \mathrm{~L}$ of water. Finally, the film was dried in a vacuum oven $\left(60^{\circ} \mathrm{C},<0.1 \mathrm{MPa}\right)$.

2.2. Chemical Stability Testing of CTAC-Doped CNT Film. For oxygen-resistance tests, doped CNT films were cut into rectangular pieces $(4 \mathrm{~mm} \times 16 \mathrm{~mm})$ and placed in glass Petri dishes and incubated at room temperature $\left(25^{\circ} \mathrm{C}\right)$ in air. The thermoelectric properties of the samples were periodically evaluated. For water-resistance tests, rectangular film samples $(4 \mathrm{~mm} \times 16 \mathrm{~mm})$ were incubated at room temperature for a specified number of days in a $50 \mathrm{~mL}$ screw tube containing $30 \mathrm{~mL}$ of water. Extracted samples were dried overnight in a vacuum oven $\left(60^{\circ} \mathrm{C},<0.1 \mathrm{MPa}\right)$ and evaluated for thermoelectric properties.

\section{Results}

There are various types and sizes of CNTs, and their organic thermoelectric properties vary greatly. In this study, we used single-walled CNTs (diameter $=1.5 \pm 0.8 \mathrm{~nm}$; metallic/ semiconducting ratio $=1: 2$ ) without purification. To clarify the doping effect of surfactants and unexpected solvent doping on the CNTs, a preliminary study was conducted whereby individual CNT dispersions were prepared using 19 common solvents (Figure S2). The dispersions were homogenized and sonicated, filtered through PTFE membranes, and dried $\left(60^{\circ} \mathrm{C},<0.1 \mathrm{MPa}, 12 \mathrm{~h}\right)$ to form buckypapers. Then, the Seebeck coefficients $(S)$ of the buckypapers were evaluated. No surfactants or other dispersing agents were added, and the resulting films were studied in air without any special sealing process such as lamination. The Seebeck coefficients at $72^{\circ} \mathrm{C}(=345 \mathrm{~K})$ are shown graphically in Figure 2 and listed in Table S1. The Seebeck coefficient of the CNT film prepared using water as the solvent was $+62.3 \pm 0.2 \mu \mathrm{V} \mathrm{K}^{-1}$, which is significantly higher than those of the films prepared using organic solvents. In general, CNTs behave like p-type semiconductors because they adsorb oxygen from the atmosphere. The Seebeck coefficients of the CNT films fabricated using organic solvents ranged from $+43.8 \pm 1.0$ to $-55.1 \pm 0.4 \mu \mathrm{V} \mathrm{K}^{-1}$. The films produced with polar protic solvents like alcohols and nonpolar or weakly polar solvents like chlorinated compounds had a positive Seebeck coefficient, whereas those produced with polar aprotic solvents, such as nitrogen-containing compounds or carbonyl compounds, had a negative Seebeck coefficient (with the exception of the film produced in dichloromethane, which had a positive Seebeck coefficient). These results indicate a clear difference between the types of charge carriers in the films obtained using different types of solvents. This is likely due to differences in the chemical species adsorbed on the CNTs following the solvent processing.

The thermogravimetric profiles of the CNT samples can provide information about the nature of the adsorption of residual solvent molecules on the CNT surfaces [35-37]. The thermograms of both p- and n-type CNT film samples are shown in Figure S3(A) and (B). The weight loss at temperatures higher than $350^{\circ} \mathrm{C}$ of the CNTs processed in aqueous media indicates the presence of defects on the walls of the nanotubes [38]. Although pristine CNTs are stable at temperatures lower than $350^{\circ} \mathrm{C}$, the CNTs processed using organic solvents exhibit a loss in weight in this temperature range, corresponding to the thermal evolution of solvent molecules adsorbed on the CNTs. The weight loss of the various samples at $350^{\circ} \mathrm{C}$ is provided in Table S1. It is important to note that the weight losses of the CNT samples processed in organic solvents at temperatures below the boiling points of the respective solvents are 


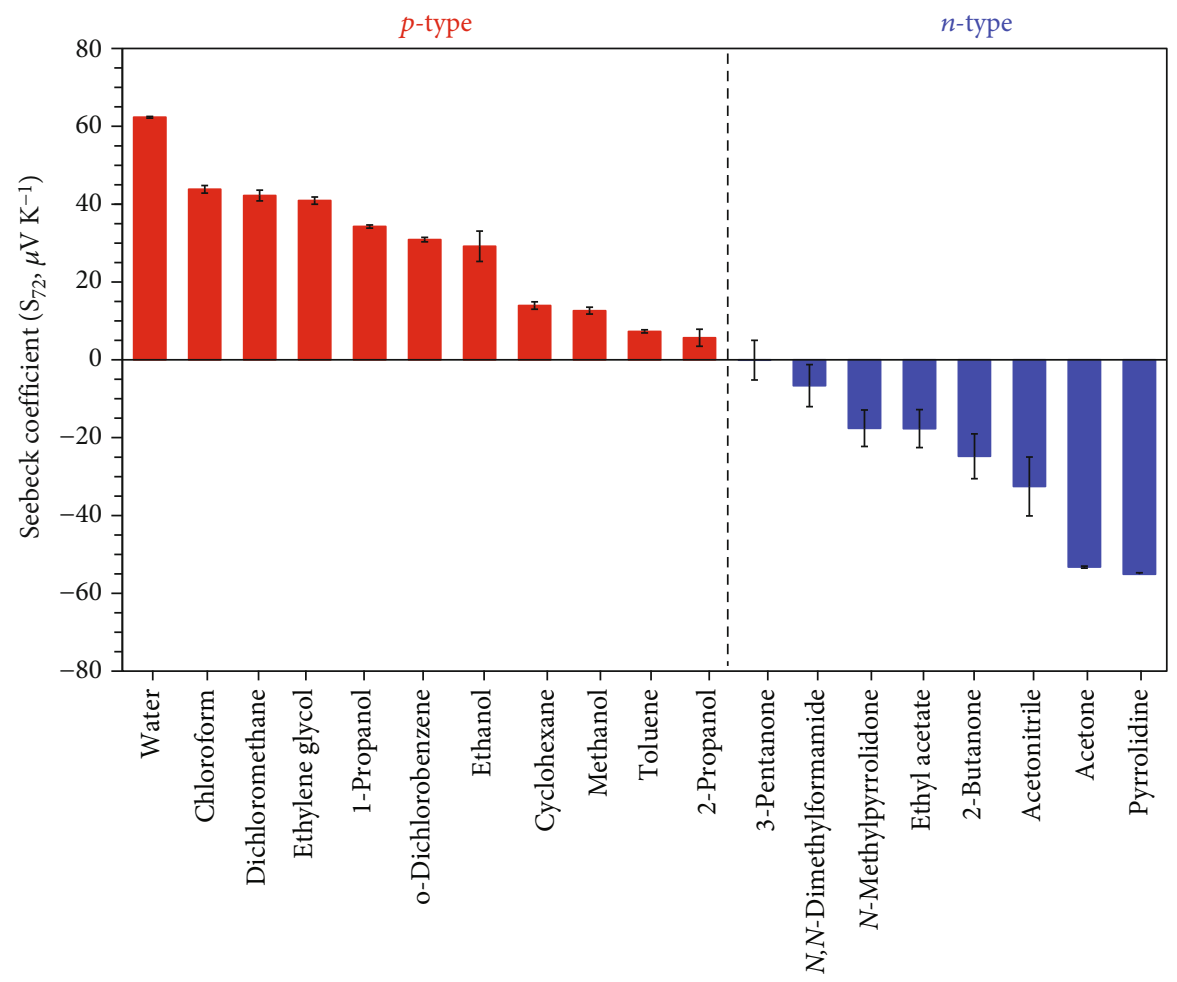

Figure 2: Effect of solvent type on Seebeck coefficient at $72^{\circ} \mathrm{C}(=345 \mathrm{~K})$ of CNT films. Red: samples with positive Seebeck coefficients; blue: samples with negative Seebeck coefficients. The CNT dispersions were homogenized and sonicated, filtered through PTFE membrane filters, and dried $\left(60^{\circ} \mathrm{C},<0.1 \mathrm{MPa}, 12 \mathrm{~h}\right)$ prior to Seebeck coefficient measurements. No dispersants or surfactants were added.

negligible (Figure S2), implying that the solvent molecules are not merely physisorbed but chemisorbed or electrostatically adsorbed on the CNTs. Collins et al. reported that the electronic properties (thermoelectric power and local density of states) of CNTs, which have a large specific surface, are very sensitive to the surrounding chemical environment [39-41]. The differences in the positive Seebeck coefficients of the p-type CNTs obtained by processing in water, alcohols, and chlorinated solvents may be caused by the inhibition of molecular oxygen adsorption by the presence of residual solvent molecules, which would reduce the oxygen doping level of the CNTs. When CNTs are processed using polar aprotic solvents like $N, N$-dimethylformamide (DMF) and $N$-methylpyrrolidone (NMP), the unshared electron pairs on the adsorbed solvent molecules are transferred to the CNTs, inducing a change in the semiconductor behavior from p- to n-type [42]. Even without an organic dopant, it is possible to induce electron donation from solvent molecules to the CNTs, i.e., dopants can have unintended n-doping effects on CNTs. These results indicate that the solvent used for CNT processing has a strong effect on the Seebeck coefficient of the processed CNTs and provide a basis for understanding the nature of CNT doping by different chemical species. The inferences drawn from this preliminary study are the basis for developing green chemistry approaches for the synthesis of n-type CNTs by doping them with cationic surfactants in aqueous media, without recourse to organic solvents.
Figure 3 summarizes the $S, \sigma$, and $\mathrm{PF}$ values of the surfactant-doped CNT films at $72^{\circ} \mathrm{C}$. The Seebeck coefficients of OTAC-, DTAC-, and CTAC-doped-CNT were $-50.9,-38.2$, and $-34.2 \mu \mathrm{VK}^{-1}$, respectively. In contrast to the pure CNT films, all the cationic surfactant-doped CNT films exhibited negative Seebeck coefficients, regardless of the length of the alkyl chain attached to the nitrogen atom in the surfactant molecules. This implies that the surfactant acts not only as a dispersant but also as a dopant. We studied various ionic surfactants with $\mathrm{C} 12$ hydrophobic tails and found that the doped CNTs are p-type when the dopant is anionic and n-type when the dopant is cationic, irrespective of the concentration of the surfactant in the aqueous solution used for dispersing the pristine CNTs (Figure S4). Countercations are essential for stabilizing negatively charged sites on the CNT wall. Thus, the ionicity of the surfactant determines the type of charge carrier in the doped CNTs. The n-doping of CNTs is caused by the adsorption of the cationic head groups of the surfactant on the CNT surface, likely causing an upward shift in its Fermi energy [43]. This phenomenon indicates a Lewis acid-base complexation between the electron-accepting ammonium head group and the electron-donating CNTs, which exhibit electron donation ability similar to other aromatic compounds $[32,44]$. Note that the absolute value of the negative Seebeck coefficient decreases as the length of the alkyl group of the surfactant increases. As the tradeoff between the electrical conductivity and Seebeck 


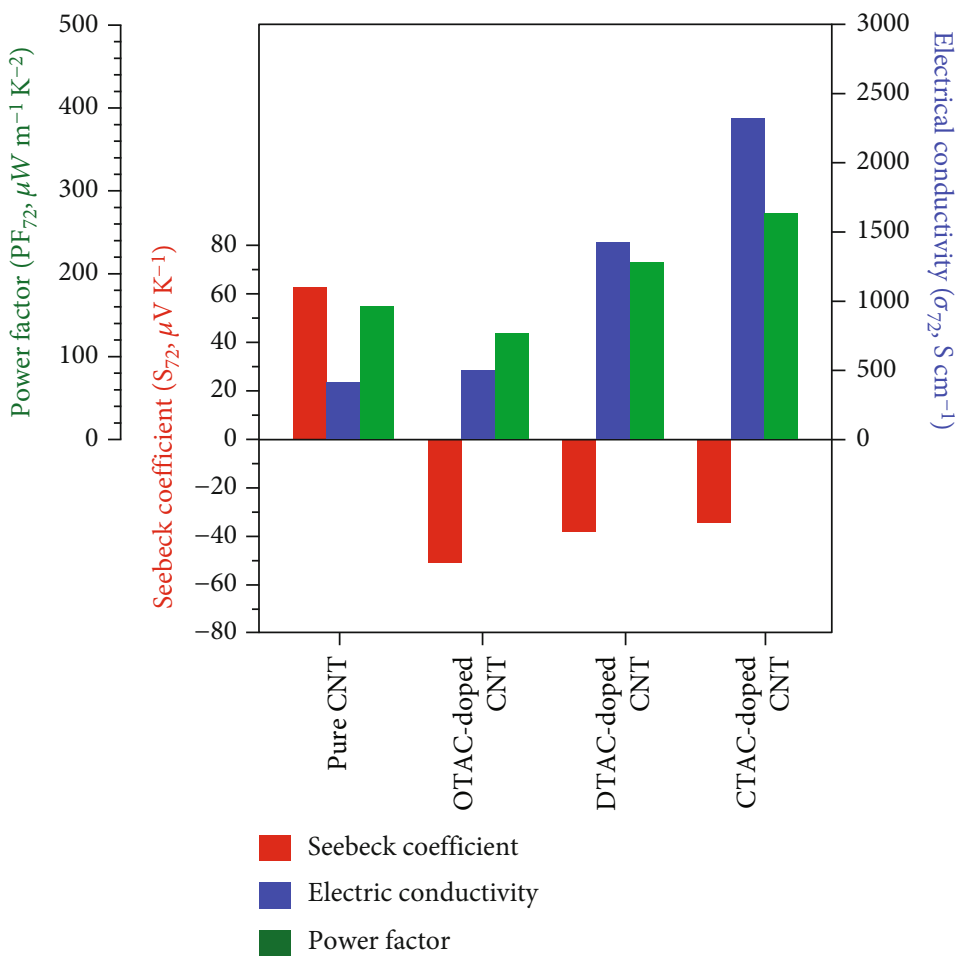

FIgURE 3: In-plane thermoelectric properties of doped CNT films prepared in aqueous media. Measurements were performed at $72^{\circ} \mathrm{C}$ in a He atmosphere.

coefficient is well known, the Seebeck coefficients were expected to be inversely correlated under the influence of electrical conductivity, which will be discussed next. The electrical conductivities $(\sigma)$ of the OTAC-, DTAC-, and CTAC-doped-CNTs were 493, 1418 , and $2316 \mathrm{~S} \mathrm{~cm}^{-1}$, respectively. Thus, the electrical conductivity increases with increasing length of the alkyl groups in the surfactants. In keeping with this trend, the PF values of the CNT films doped with surfactants vary in the order CTAC $>$ DTAC $>$ OTAC.

Table S2 summarizes the thermoelectric properties of recently reported n-type CNTs obtained by chemical doping $[28,29,43,45-49]$. In comparison, the CTAC-doped CNTs exhibited an excellent $\mathrm{PF}$ value $\left(271 \mu \mathrm{V} \mathrm{m}^{-1} \mathrm{~K}^{-2}\right)$, even though their synthesis did not require organic solvents, strongly alkaline aqueous solutions, high concentrations of dopants in the solvents, or long doping times, unlike the ntype CNTs presented in Table S2. Water-processable n-type CNTs typically have a PF of around $200 \mu \mathrm{Vm}^{-1} \mathrm{~K}^{-2}$ $[33,43]$; however, they are usually prepared by treating a p-type CNT film with a water-soluble n-dopant, which is not necessary in the present experiment. Doping by the most effective dopant reported, cetyltrimethylammonium bromide (CTAB), yields a PF value of only $210 \mu \mathrm{V} \mathrm{m}^{-1} \mathrm{~K}^{-2}$ [50]. To the best of our knowledge, this is the first report to show that $\mathrm{n}$-type CNTs that are superior to conventional systems can be prepared in aqueous media using appropriate cationic surfactants. As shown in Table S2, the electrical conductivity of the CTAC-doped CNTs is one order of magnitude higher than those of most previously reported doped CNTs. To elucidate the reason for this observation, the pristine and doped CNT films were studied by high-resolution scanning electron microscopy (SEM) (Figures 4(a)-4(d)). Pristine CNTs aggregate into ropes or bundles owing to strong van der Waals forces and $\pi-\pi$ interactions between the CNT walls [51]. The diameters of the bundles had a wide distribution of $41.8 \pm 33.7 \mathrm{~nm}$ (Figure 4(a)). The diameters of the CNT bundles in the OTAC-, DTAC-, and CTACdoped CNT films were $36.5 \pm 32.9,34.0 \pm 27.9$, and $23.6 \pm$ $22.1 \mathrm{~nm}$, respectively, indicating that the surfactants induced exfoliation of the CNTs.

From the powder X-ray diffraction pattern data, it was found that the $2 \theta$ value of the (002) graphite peak of CNTs was downshifted according to the alkyl chain length of the surfactant used (Figure S5(A)). The interlayer distance of the graphite (002) planes was widest when CTAC molecules were intercalated into the nanotube bundle [52]. At the same time, it was confirmed that there were no surfactant-derived crystals in the prepared film (Figure S5(B)). The presence of dopant molecules was confirmed by X-ray photoelectron spectroscopy (XPS). The XPS $\mathrm{N} 1 \mathrm{~s}$ region scans of the pristine and surfactant-doped CNTs (Figure S6) revealed that the longer the alkyl chain in the surfactant molecule, the greater the amount of surfactant adsorbed on the CNTs. This evidence suggests that the surfactant is present in molecular form in the CNT films. Overall, the morphology of the CNTs, as revealed by the SEM images, indicates exfoliation of the CNT bundles in the aqueous surfactant solution, enabled by the adsorption surfactants on the hydrophobic 


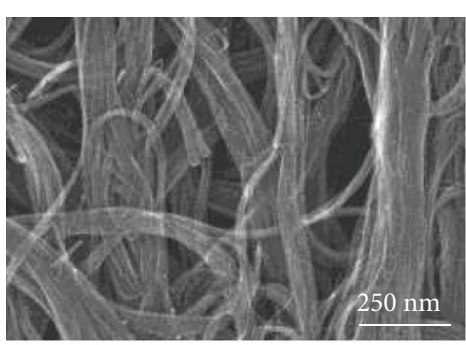

(a)

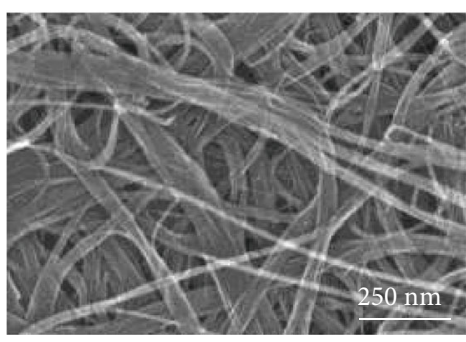

(c)

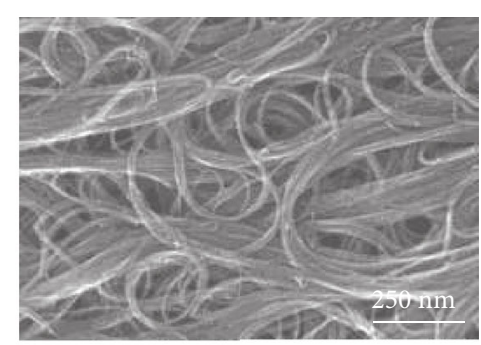

(b)

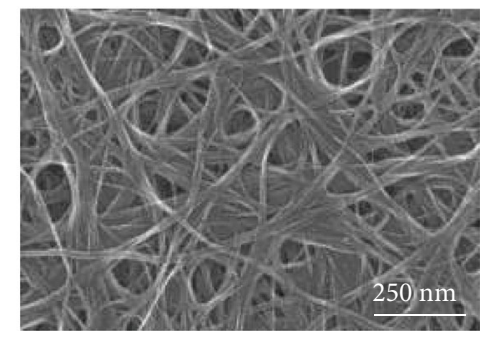

(d)

FIgure 4: SEM images of (a) pristine and (b) OTAC-, (c) DTAC-, and (d) CTAC-doped CNT films.

CNT surfaces, which renders the CNT surfaces hydrophilic and makes them easier to disperse [53, 54]. In particular, CTAC molecules have been shown to weaken the chemical interactions between CNT tubes, resulting in the exfoliation of CNT ropes, which increases the number of conduction pathways available for charge transport in the CNT films [55].

Water-vapor adsorption isotherms (Figure 5(a)) were acquired to examine the surface properties of the CNT films. The isotherms are S-shaped, with significant water vapor adsorption observed above a relative pressure $\left(P / P_{0}\right)$ of 0.5 . Importantly, the surfactant-doped CNTs adsorbed significantly higher amounts of water than the pristine CNTs, especially at lower relative pressures $\left(0<P / P_{0}<0.4\right)$. In this regard, the apparent $\mathrm{H}_{2} \mathrm{O}$ specific surface areas of the pristine and OTAC-, DTAC-, and CTAC-doped CNTs were determined by the Brunauer-Emmett-Teller (BET) method to be $14,49,103$, and $120 \mathrm{~m}^{2} \mathrm{~g}^{-1}$, respectively (Figure 5(b)), which indicates that the surfactant-doped CNTs had higher affinity for water owing to the surfactant molecules adsorbed on the walls of the nanotubes. Further, the order of the BET surface areas is consistent with that of the bundle diameters shown by the SEM images, suggesting that the longer the alkyl chain in the surfactant molecule, the greater the amount of surfactant adsorbed on the nanotubes and the better the exfoliation. Therefore, the use of CTAC, which has the longest alkyl chain of the surfactants used, may have resulted in better dispersion of the CNTs in water. In order to further confirm the results, we measured the $\mathrm{N}_{2}$ adsorption isotherms at $-196^{\circ} \mathrm{C}$. The plot between the specific surface area, obtained using the BET method for each surfactant-doped CNT film, and the apparent surfactant content in the film determined using TG measurement is shown in Figure S7. The $\mathrm{N}_{2}$ specific surface areas of the nanotubes decreased with the tail length of the surfactant molecules, which implies that the adsorbed surfactant eliminates the mutual force between the CNT surface and the adsorbed $\mathrm{N}_{2}$. It is important to note that the surface areas of the bare nanotube in the CNT film tend to decrease as the amount of surfactant in the film increases. Therefore, the longer the tail of the dispersed surfactant, the greater the amount of surfactant remaining in the film.

The chemical interactions and possible charge transfer at the nanotube/surfactant interfaces of the doped CNT samples were studied by Raman spectroscopy (Figure 5(c)). The G-bands can be seen at 1589, 1590, 1591, and $1592 \mathrm{~cm}^{-1}$ for the pristine and OTAC-, DTAC-, and CTAC-doped CNTs, respectively. The shift in the G-band wavenumber is proportional to the length of the alkyl chain of the surfactant. The blue shift of the G-band was predicted to depend on the coverage of the surfactant molecule, and it indicates that the surfactant molecules donate electrons to the CNTs. Such a shift in the position of the G-band has often been observed for CNTs coated with amphiphilic polycations [56, 57]. Therefore, the Raman spectra suggest that the surfactant molecules are not merely physisorbed on the CNT surfaces but contribute to charge transfer to the CNTs. The creation of defects on the CNT backbone as a result of chemical treatment can be assessed from the ratio of the area under the D-band, which is related to structural defects, to that under the G-band, which is related to the graphitic structure of the nanotubes [51]. No noticeable changes in the $D / G$ ratio can be observed in the spectra of the surfactant-doped CNTs compared to that of the pristine CNTs, indicating that no significant defects or new functional groups have been introduced on the nanotube walls. This implies that the interaction between the cationic surfactants and the CNTs is noncovalent in nature. Figure S8 shows the low-frequency radial breathing modes (RBM) of the surfactant-doped CNTs. The intensity of the RBMs is reduced by surfactant-doping, as the adsorption of 


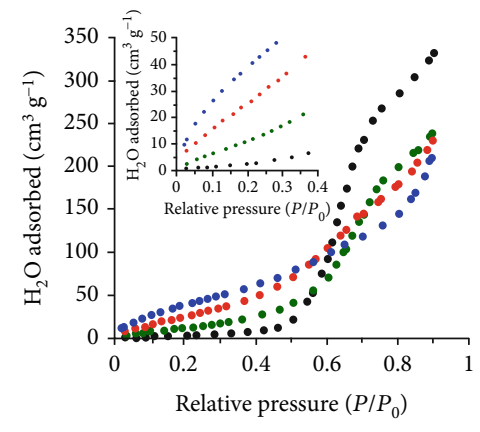

(a)

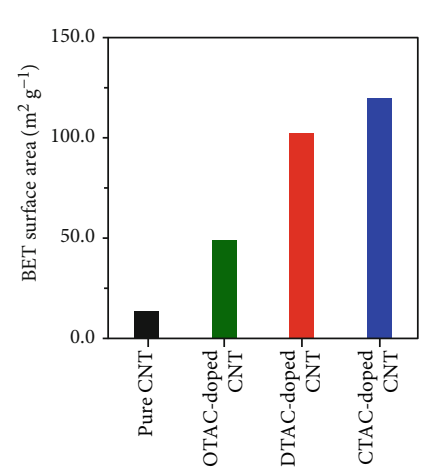

(b)

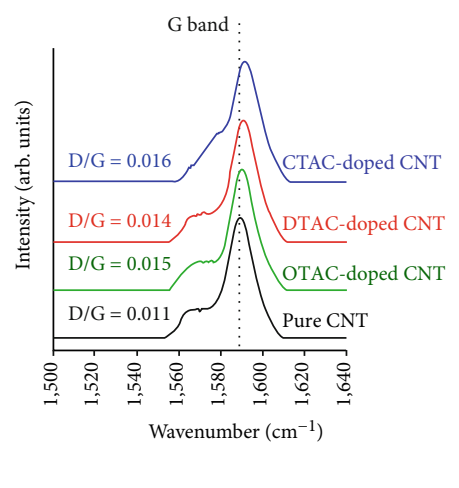

(c)

FIGURE 5: (a) Water vapor adsorption isotherms at $25^{\circ} \mathrm{C}$, (b) specific surface areas obtained from BET theory applied to the experimental isotherms, and (c) G-bands in the Raman spectra. Black: CNT film; green: OTAC-doped CNT film; red: DTAC-doped CNT film; blue: CTAC-doped CNT film.

surfactant molecules on the CNT walls hinders the expansion/contraction vibration of the nanotubes [58]. This observation is consistent with surfactant-wrapping, as has also been observed for CNTs coated with PEI [28].

It remains a challenge to synthesize CNT-based thermoelectric materials using stable n-type doped CNTs that are not sensitive to atmospheric oxygen and in which the dopants do not undergo degradation. The stability of the CTAC-doped CNTs was evaluated by exposing them to air and measuring their properties after various intervals of time (Figure 6(a)). The initial values of $S$ and PF for CTAC-doped CNTs are $-34.2 \mu \mathrm{V} \mathrm{K}^{-1}$ and $271 \mu \mathrm{V} \mathrm{m}^{-1} \mathrm{~K}^{-2}$, respectively, and showed very little variation over a period of 185 days. These results indicate that the thermoelectric performance of CTAC-doped CNT showed no significant degradation in air. The stability of our CTAC-doped CNTs is comparable with that of n-type CNTs obtained by doping with 2-aryl1,3-dimethyl-2,3-dihydro-1H-benzo[d]imidazole [47] and superior to that of n-type CNTs in most other previous reports (Table S2).

To evaluate dedoping, the doped CNT films were stored in water, and their thermoelectric properties measured at various intervals over a period of 28 days (Figure 6(b)). For reference, CNTs doped with $\mathrm{NaBH}_{4}$ and PEI were also subjected to identical testing (Figure S9). The values of $S$ and PF for the CTAC-doped CNTs were nearly constant over the testing period, implying that the CTAC molecules were stably anchored to the surface of the nanotubes, and their adsorption was not reversed by immersion in water. The $\mathrm{NaBH}_{4}$-doped CNTs switched from n- to p-type behavior after 7 days of immersion in water, while the Seebeck coefficient for PEI-doped CNTs was nearly constant over 28 days. This suggests that the $\mathrm{NaBH}_{4}$-doped CNTs lack a protective layer to shield the CNTs from attack by $\mathrm{H}_{2} \mathrm{O}$ molecules. On the other hand, the PEIdoped CNTs were shielded by the PEI chains wrapped around the CNTs. The similarity in the stability of our CTAC-doped CNTs with those of PEI-doped CNTs suggests that the CTAC molecules are wrapped around the nanotubes, forming a protective layer (Figures 7 (a) and $7(\mathrm{~b}))$. It is worth emphasizing that the wrapping of the cationic electron cloud inhibits direct contact of molecular oxygen and water with the CNTs, thus, preserving the electron carrier pathway. The low stability of n-type organic semiconductors in the presence of water has been a major concern for a long time $[59,60]$. We have addressed this problem by optimizing the interface between the surfactant and the CNTs, as a result of which stable n-type characteristics-that were not reversed even when the material was submerged in water for a prolonged period-were induced.

We created modules incorporating CTAC-doped CNTs (n-type) and sodium dodecylbenzenesulfonate- (SDBS-) doped CNTs (p-type). The detailed thermophysical properties and $Z T$ values of the $\mathrm{p}$ - and n-materials used in the module configuration are summarized in Table S3. Five $\mathrm{p}$ - and n-type films, cut to a length of $6 \mathrm{~cm}$ and width of $0.5 \mathrm{~cm}$, were alternately laid out on a polyimide substrate. The films were connected in series using Ag paste electrodes to produce a 5 -unit $\mathrm{p}-\mathrm{n}$ device (Figure $8(\mathrm{a})$ ). Figure S10(A) shows a schematic of the module evaluation. The hot end of the device was heated on a heating plate, and the cold end was exposed to air. The temperatures of the hot $\left(T_{\text {hot }}\right)$ and cold $\left(T_{\text {cold }}\right)$ ends were monitored using data acquisition and a logging multimeter system. The temperature difference $\Delta T\left(=T_{\text {hot }}-T_{\text {cold }}\right)$ was controlled by regulating the temperature of the heating plate. Figure 8(b) shows the voltage generated for each value of $\Delta T$. The theoretical voltage $\left(V_{\mathrm{TH}}\right)$ was calculated as follows [61]:

$$
V_{\mathrm{TH}}=\left(N_{p} S_{p}+N_{n} S_{n}\right) \Delta T
$$

where $N$ and $S$ are the number of units and Seebeck coefficient of the corresponding $\mathrm{p}$ - and n-type CNT films, respectively. The actual voltage $\left(V_{\mathrm{AC}}\right)$ of the fabricated device shows a good linear relationship with $\Delta$ $T$, and its value is very close to that of $V_{\mathrm{TH}}$. The output power-output current and output voltage-output current curves of the CNT base module at $\Delta T=15,30,45,60$, and $75^{\circ} \mathrm{C}$ are shown in Figure $8(\mathrm{c})$. The output power- 


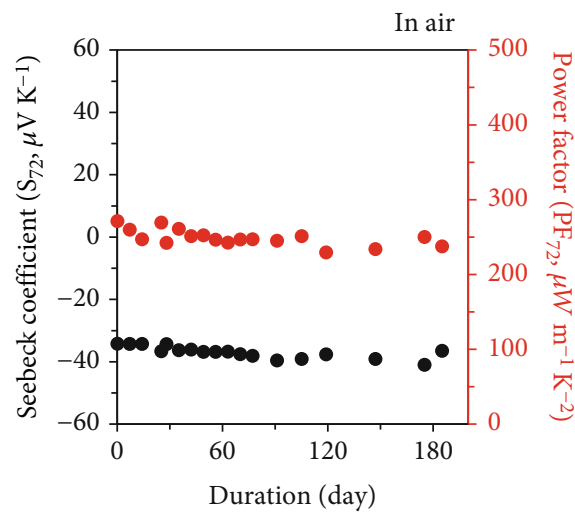

(a)

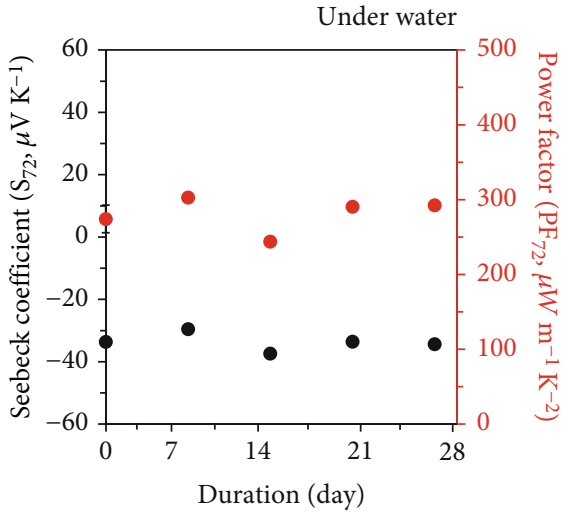

(b)

FIGURE 6: Variation in Seebeck coefficient (black circles) and thermoelectric power factor (red circles) of CTAC-doped CNT films with duration of exposure to (a) air and (b) water. Measurements were performed at $72^{\circ} \mathrm{C}$ in $\mathrm{He}$.

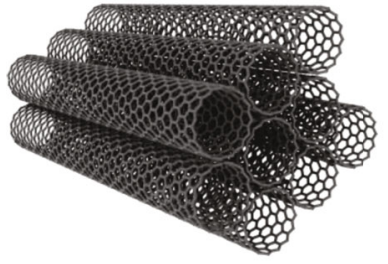

Carbon nanotube bundle

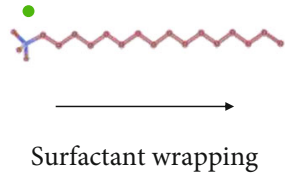

(a)
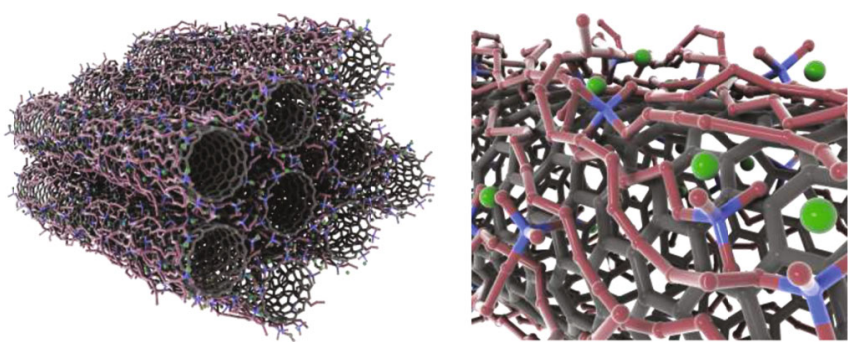

(b)

FIgURE 7: (a) Schematic showing the CNT bundle wrapped by surfactant molecules. (b) Adsorption-layer of CTAC molecules on a nanotube. Blue: $\mathrm{N}$ atoms; brown: $\mathrm{C}$ atoms; green: $\mathrm{Cl}$ atoms.

output current curve was parabolic, and the maximum output powers $\left(P_{\mathrm{AC}}\right)$ at $\Delta T=15,30,45,60$, and $75^{\circ} \mathrm{C}$ were approximately $0.24,0.75,2.23,3.40$, and $6.32 \mu \mathrm{W}$, respectively. The maximum power density of this device was found to be $117 \mu \mathrm{W} \mathrm{g}^{-1}$ at $\Delta T=75^{\circ} \mathrm{C}$. The theoretical output power $\left(P_{\mathrm{TH}}\right)$ was calculated as follows [49]:

$$
P_{\mathrm{TH}}=\frac{V_{\mathrm{TH}}^{2}}{4 R_{i}},
$$

where $R_{i}$ is the internal resistance of the fabricated $\mathrm{p}-\mathrm{n}$ device $(16.3 \Omega)$. The results show that the internal resistance of these modules is relatively low compared to those of previously reported organic thermoelectric modules $[61,62]$. The $P_{\mathrm{AC}}$ values for $\Delta T=15,30,45,60$, and $75^{\circ} \mathrm{C}$ compare favorably with the $P_{\mathrm{TH}}$ values calculated using Equation (4) (Figure S10(B)), suggesting that higher power output can be achieved by connecting more units in series. The waterresistance of the CTAC-doped CNTs incorporated in the modules was also investigated. The unsealed module was immersed in $900 \mathrm{~mL}$ water as shown in Figure 9(a). The modules were then removed after intervals of 5 days, dried overnight in a vacuum oven $\left(60^{\circ} \mathrm{C},<0.1 \mathrm{MPa}\right)$, and the output of the modules $\left(\Delta T=45\right.$ and $\left.75^{\circ} \mathrm{C}\right)$ was measured (Figure $9(\mathrm{~b})$ ). The total duration of the test was 30 days.
The decrease in the module output over the duration of the test is low at both temperature gradients $(20 \pm 2.4 \%$ for $\Delta T=75^{\circ} \mathrm{C}$ ), indicating that the power generation performance of the device is stable and the CTAC-doped $\mathrm{CNTs}$ in the module are water-resistant. In recent reports of soft flexible electronics for underwater applications like organic solar cells [19], bionic stretchable nanogenerators [63], and tribo-ferroelectric electronic textiles [18], water resistance was ensured by coating the generators with polymer films or carbon fibers. This additional waterproofing layer is not necessary for our thermoelectric power generation module based on surfactant-doped CNTs. The bare organic thermoelectric modules are capable of providing stable power on the order of microwatts after submergence in water for several days, implying that they can be reliably used for energy recovery and reuse in underwater applications.

\section{Discussion}

Measurement of the Seebeck coefficients of surfactant-free CNT films prepared using 19 commonly used organic solvents indicated that the carrier properties of the CNTs were considerably affected by the residual solvent adsorbed on the CNT surfaces. CNTs exhibited p-type behavior when processed in polar protic or nonpolar chlorinated solvents and 


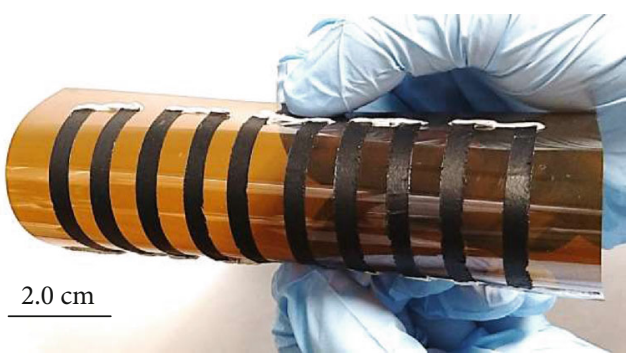

(a)

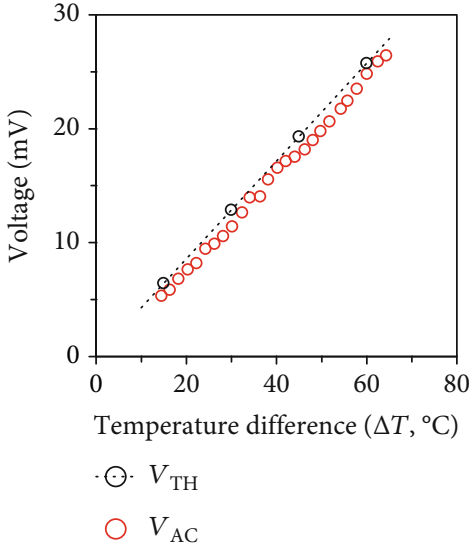

(b)

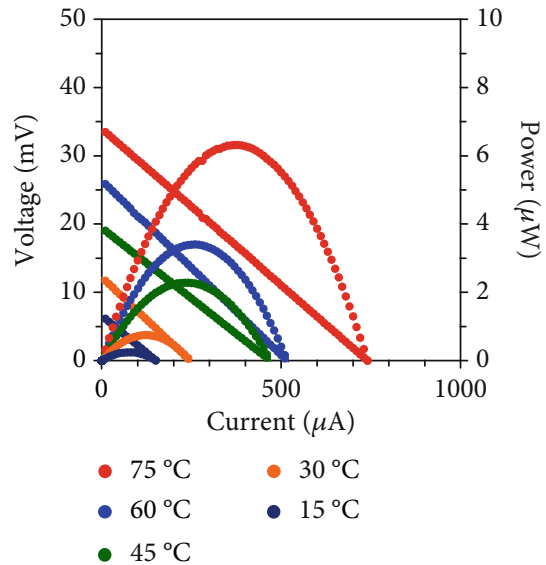

(c)

FIGURE 8: (a) Digital photograph of the 5-unit p-n device showing good flexibility. (b) Voltage generated as a function of the steady-state temperature difference between the two ends of the device, where $V_{\mathrm{TH}}$ is the theoretical voltage and $V_{\mathrm{AC}}$ is the actual voltage of the assembled device. (c) Output power-output current and output voltage-output current curves of the device at temperature differences of $15,30,45,60$, and $75^{\circ} \mathrm{C}$ between the two ends.

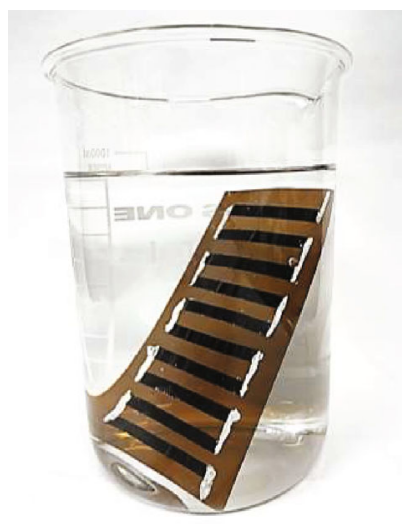

(a)

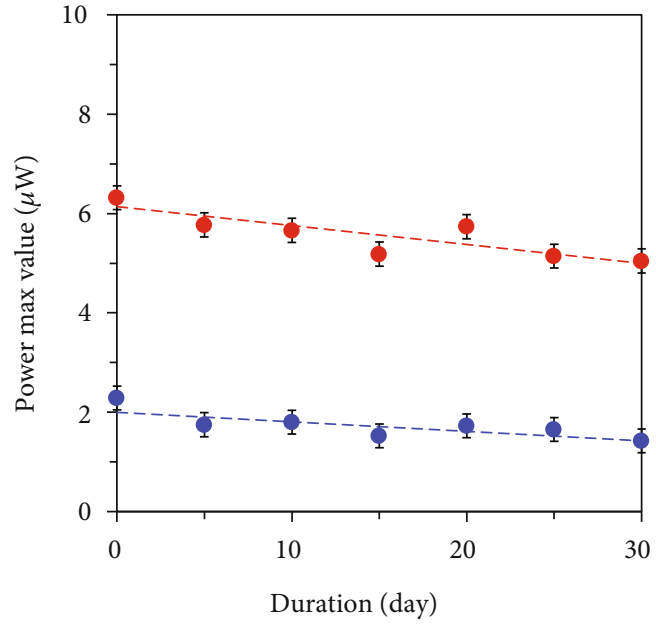

- $75^{\circ} \mathrm{C}$

- $45{ }^{\circ} \mathrm{C}$

Figure 9: (a) Water submergence test of 5-unit $\mathrm{p}-\mathrm{n}$ device. (b) Variation in maximum output power of the device with duration of submergence underwater, for temperature differences of 45 and $75^{\circ} \mathrm{C}$ between the hot and cold ends.

n-type behavior when processed in polar aprotic solvents. The CNT film prepared using water had the highest value of $S\left(+62.3 \pm 0.2 \mu \mathrm{V} \mathrm{K}^{-1}\right)$. We found that cationic surfactants are suitable dopants for CNTs to induce a change from $\mathrm{p}$ - to $\mathrm{n}$-type behavior. In general, it is difficult to fabricate n-type CNT membranes using aqueous solutions because the 
electrophilic reaction between the electron activation sites and water molecules causes the doping level and Seebeck coefficient of CNTs with n-type doping to revert to the airexposed state. However, the cationic head groups of surfactants can induce n-type behavior in CNTs, even when using aqueous media. The extent of exfoliation of the CNT bundles, and concomitantly, the electrical conductivity, and thermoelectric PF of the CNT films increase with increasing surfactant tail length.

Ethanol-containing polyethyleneimine has excellent PF values with high electrical conductivity, as summarized in Table S2. This is because the host material is a CNT film deposited in situ by chemical vapor deposition, which provides good contact at the intertube junctions. A common method for fabricating CNT films is by filtering dispersed CNT solutions (filter-deposited buckypaper), whereby the contact between nanotube bundles is weak and blocked by insulating surfactant residues or n-type dopants. This process unfortunately creates an insulating barrier between CNT bundles, resulting in high resistance between the CNTs and an electrical conductivity of less than $1000 \mathrm{~S} \mathrm{~cm}^{-1}$. In contrast, the proposed method, whereby CNT films are prepared using an aqueous solution of a cationic surfactant with a long alkyl chain, is expected to reduce the bundle size of the random CNT network and improve the contact at intertube junctions, as the excess dopant agent is washed away. In other words, the green route using surfactant solution in this work is an optimal strategy for increasing the n-type PF value and electrical conductivity, since sufficient washing removes the excess surfactant without dedoping through strong chemical interaction between the surfactant hydrophilic group and the nanotubes. To fully oxidize the samples, we immersed them in water for a long time. The thermoelectric properties of the immersed films showed no significant changes for at least 28 days, demonstrating that the $\mathrm{n}$-doped CNTs are stable underwater. We infer that the adsorption layer of surfactant molecules acts as a barrier layer to maintain air stability for a long time. It is speculated that this is because the negatively charged doping sites are less sensitive to oxygen and water. Although n-type CNTs have been extensively studied over the past few decades, their electrical conductivity and poor chemical stability, as well as the lack of inexpensive and lowwaste methods in liquid-phase doping, have remained a challenge. The proposed method has the potential to address the current concerns of n-type materials from preparation to characterization and provides a strategy that can lead to future industrial applications of water-treatable n-type thermoelectric membranes for advancing energy and fuel conversion. A thermoelectric power generation module comprising CTAC- and SDBS-doped CNTs was able to generate power underwater in the order of microwatts for 30 days. To the best of our knowledge, there are no reports of water-treatable flexible organic TEG, and hence, our results constitute a milestone.

In this study, CNTs with relaxed semiconducting and metallic components were used; however, the use of semiconducting CNTs with high Seebeck coefficients can accelerate the study of physical properties of thermoelectric materials and should be explored in the future. Additionally, CNTs produced via suspended chemical vapor deposition in a catalytic atmosphere or by directly forming fiber/yarn have high electrical conductivity and p-type PF due to the increased longitudinal carrier mobility because of their highly ordered structure. The present study suggests that the impregnation of CNT yarns in dilute CTAC aqueous solutions will also facilitate the synthesis of water-treatable n-type materials with high conductivity. The results of this study are not limited to films only and can also be expanded to flexible and moldable CNT forms, such as fibers and mats. Our green approach for the synthesis of water-resistant ntype CNTs may provide important insights for future endeavors for the development of organic thermoelectric materials for efficient and sustainable energy conversion.

\section{Data Availability}

The data that support the findings of this study are available from the corresponding authors upon reasonable request.

\section{Conflicts of Interest}

The authors declare that there is no conflict of interest regarding the publication of this article.

\section{Authors' Contributions}

S.H. and Y.S. conceived the original concept and designed the experiments. S.H. wrote the manuscript. S.H., M.S., and S.Y. performed the material synthesis and characterization. G.J, Y.D., and N.T. gave advice about the interpretation of the results. S.H. and Y.S. supervised this project. All authors contributed to discuss the results.

\section{Acknowledgments}

This study was supported in part by the projects JSPS KAKENHI (nos. $21 \mathrm{~K} 14428$ to S.H. and 19K05633 to Y.S.), Foundation from Oil \& Fat Industry Kaikan, and the Environmental research grant from Steel Foundation for Environmental Protection Technology, Japan.

\section{Supplementary Materials}

Experimental overview, characterization, TG-DTA data, photograph of fabricated film, thermoelectric properties of various surfactant-doped CNT, XRD data, XPS data, RBM region from the Raman spectra, thermoelectric properties of $\mathrm{NaBH}_{4}$-doped $\mathrm{CNT}$ and PEI-doped CNT, apparent hydrophobic/hydrophilic ratio of the CTAC-doped CNT and SDS-doped CNT, thermoelectric conversion characteristics and thermophysical properties of the CTAC-doped CNT and SDBS-doped CNT, and evaluation of fabricated device. (Supplementary Materials)

\section{References}

[1] M. S. Bhatt, "Energy audit case studies I-steam systems," Applied Thermal Engineering, vol. 20, pp. 285-296, 2000. 
[2] D. B. Gingerich and M. S. Mauter, "Quantity, quality, and availability of waste heat from United States thermal power generation," Environmental Science \& Technology, vol. 49, pp. 8297-8306, 2015.

[3] A. P. Raman, M. A. Anoma, L. Zhu, E. Rephaeli, and S. Fan, "Passive radiative cooling below ambient air temperature under direct sunlight," Nature, vol. 515, pp. 540-544, 2014.

[4] S. W. Lee, Y. Yang, H.-W. Lee et al., "An electrochemical system for efficiently harvesting low-grade heat energy," Nature Communications, vol. 5, p. 3942, 2014.

[5] G. J. Snyder and E. S. Toberer, "Complex thermoelectric materials," Nature Materials, vol. 7, pp. 105-114, 2008.

[6] H. Yan and N. Toshima, "Thermoelectric properties of alternatively layered films of polyaniline and (+/-)-10-camphorsulfonic acid-doped polyaniline," Chemistry Letters, vol. 28, pp. 1217-1218, 1999.

[7] N. Toshima, "Recent progress of organic and hybrid thermoelectric materials," Synthetic Metals, vol. 225, pp. 3-21, 2017.

[8] X. Huang, L. Deng, F. Liu, Q. Zhang, and G. Chen, "Effect of crystalline microstructure evolution on thermoelectric performance of PEDOT: PSS films," Energy Material Advances, vol. 2021, article 1572537, 10 pages, 2021.

[9] Y. Hiroshige, M. Ookawa, and N. Toshima, "Thermoelectric figure-of-merit of iodine-doped copolymer of phenylenevinylene with dialkoxyphenylenevinylene," Synthetic Metals, vol. 157, pp. 467-474, 2007.

[10] J. Wu, Y. Sun, W. Xu, and Q. Zhang, "Investigating thermoelectric properties of doped polyaniline nanowires," Synthetic Metals, vol. 189, pp. 177-182, 2014.

[11] Y. Zhang, Q. Zhang, and G. Chen, "Carbon and carbon composites for thermoelectric applications, carbon," Energy, vol. 2, pp. 408-436, 2020.

[12] C.-J. Yao, H.-L. Zhang, and Q. Zhang, "Recent progress in thermoelectric materials based on conjugated polymers," Polymers, vol. 11, p. 107, 2019.

[13] W. Gao, H. Ota, D. Kiriya, K. Takei, and A. Javey, "Flexible electronics toward wearable sensing," Accounts of Chemical Research, vol. 52, pp. 523-533, 2019.

[14] J. Heikenfeld, A. Jajack, J. Rogers et al., "Wearable sensors: modalities, challenges, and prospects," Lab on a Chip, vol. 18, pp. 217-248, 2018.

[15] D. Son, J. Lee, S. Qiao et al., "Multifunctional wearable devices for diagnosis and therapy of movement disorders," Nature Nanotechnology, vol. 9, pp. 397-404, 2014.

[16] J.-H. Bahk, H. Fang, K. Yazawa, and A. Shakouri, "Flexible thermoelectric materials and device optimization for wearable energy harvesting," Journal of Materials Chemistry C, vol. 3, pp. 10362-10374, 2015.

[17] S. Patel, H. Park, P. Bonato, L. Chan, and M. Rodgers, "A review of wearable sensors and systems with application in rehabilitation," Journal of NeuroEngineering and Rehabilitation, vol. 9, p. 21, 2012.

[18] W. Yang, W. Gong, C. Hou et al., "All-fiber tribo-ferroelectric synergistic electronics with high thermal-moisture stability and comfortability," Nature Communications, vol. 10, p. $5541,2019$.

[19] H. Jinno, K. Fukuda, X. Xu et al., "Stretchable and waterproof elastomer-coated organic photovoltaics for washable electronic textile applications," Nature Energy, vol. 2, pp. 780$785,2017$.
[20] W.-J. Song, S. Lee, G. Song et al., "Recent progress in aqueous based flexible energy storage devices," Energy Storage Materials, vol. 30, pp. 260-286, 2020.

[21] C. Yu, K. Choi, L. Yin, and J. C. Grunlan, "Light-weight flexible carbon nanotube based organic composites with large thermoelectric power factors," ACS Nano, vol. 5, pp. 7885-7892, 2011.

[22] C. Meng, C. Liu, and S. Fan, "A promising approach to enhanced thermoelectric properties using carbon nanotube networks," Advanced Materials, vol. 22, pp. 535-539, 2010.

[23] J. L. Blackburn, A. J. Ferguson, C. Cho, and J. C. Grunlan, "Carbon-nanotube-based thermoelectric materials and devices," Advanced Materials, vol. 30, p. 1704386, 2018.

[24] N. Toshima, K. Oshima, H. Anno et al., "Novel hybrid organic thermoelectric materials: three-component hybrid films consisting of a nanoparticle polymer complex, carbon nanotubes, and vinyl polymer," Advanced Materials, vol. 27, pp. 22462251, 2015.

[25] G. D. Mahan, "Good thermoelectrics," Solid-State Physics, vol. 51, pp. 81-157, 1997.

[26] C. Yu, A. Murali, K. Choi, and Y. Ryu, "Air-stable fabric thermoelectric modules made of $\mathrm{N}$ - and P-type carbon nanotubes," Energy \& Environmental Science, vol. 5, pp. 94819486, 2012.

[27] D. D. Freeman, K. Choi, and C. Yu, "N-type thermoelectric performance of functionalized carbon nanotube-filled polymer composites," PLoS One, vol. 7, article e47822, 2012.

[28] W. Zhou, Q. Fan, Q. Zhang et al., "High-performance and compact-designed flexible thermoelectric modules enabled by a reticulate carbon nanotube architecture," Nature Communications, vol. 8, p. 14886, 2017.

[29] X. Cheng, X. Wang, and G. Chen, "A convenient and highly tunable way to n-type carbon nanotube thermoelectric composite film using common alkylammonium cationic surfactant," Journal of Materials Chemistry A, vol. 6, pp. 1903019037, 2018.

[30] Y. Nonoguchi, M. Nakano, T. Murayama et al., "Simple saltcoordinated n-type nanocarbon materials stable in air," Advanced Functional Materials, vol. 26, pp. 3021-3028, 2016.

[31] D. Abol-Fotouh, B. Dörling, O. Zapata-Arteaga et al., "Farming thermoelectric paper," Energy \& Environmental Science, vol. 12, pp. 716-726, 2019.

[32] Y. Nakashima, R. Yamaguchi, F. Toshimitsu et al., "Air-stable n-type single-walled carbon nanotubes doped with benzimidazole derivatives for thermoelectric conversion and their airstable mechanism," ACS Applied Nano Materials, vol. 2, pp. 4703-4710, 2019.

[33] S. Horike, Q. Wei, K. Kirihara, and M. Mukaida, "Water-processable n-type doping of carbon nanotubes via charge transfer with imidazolium chloride salt," Chemical Physics Letters, vol. 755, article 137801, 2020.

[34] Y. Seki, K. Nagata, and M. Takashiri, "Facile preparation of air-stable n-type thermoelectric single-wall carbon nanotube films with anionic surfactants," Scientific Reports, vol. 10, p. 8104, 2020.

[35] S. A. Hodge, M. K. Bayazit, K. S. Coleman, and M. S. Shaffer, "Unweaving the rainbow: a review of the relationship between single-walled carbon nanotube molecular structures and their chemical reactivity," Chemical Society Reviews, vol. 41, pp. 4409-4429, 2012.

[36] H. C. Yau, M. K. Bayazit, J. H. Steinke, and M. S. Shaffer, "Sonochemical degradation of N-methylpyrrolidone and its 
influence on single walled carbon nanotube dispersion," Chemical Communications, vol. 51, pp. 16621-16624, 2015.

[37] Y. Nakashima, N. Nakashima, and T. Fujigaya, "Development of air-stable n-type single-walled carbon nanotubes by doping with 2-(2-methoxyphenyl)-1, 3-dimethyl-2,3-dihydro-1Hbenzo[d]imidazole and their thermoelectric properties," Synthetic Metals, vol. 225, pp. 76-80, 2017.

[38] S. Hata, M. Kusada, S. Yasuda, Y. Du, Y. Shiraishi, and N. Toshima, "Enhancement of p-type thermoelectric power factor by low-temperature calcination in carbon nanotube thermoelectric films containing cyclodextrin polymer and Pd," Applied Physics Letters, vol. 118, article 243904, 2021.

[39] P. G. Collins, K. Bradley, M. Ishigami, and A. Zettl, "Extreme oxygen sensitivity of electronic properties of carbon nanotubes," Science, vol. 287, pp. 1801-1804, 2000.

[40] J. Kong, N. R. Franklin, C. Zhou et al., "Nanotube molecular wires as chemical sensors," Science, vol. 287, pp. 622-625, 2000.

[41] V. Schroeder, S. Savagatrup, M. He, S. Lin, and T. M. Swager, "Carbon nanotube chemical sensors," Chemical Reviews, vol. 119, pp. 599-663, 2019.

[42] G. Wu, C. Gao, G. Chen, X. Wang, and H. Wang, "High-performance organic thermoelectric modules based on flexible films of a novel n-type single-walled carbon nanotube," Journal of Materials Chemistry A, vol. 4, pp. 14187-14193, 2016.

[43] Y. Nonoguchi, A. Tani, T. Ikeda et al., "Water-processable, airstable organic nanoparticle-carbon nanotube nanocomposites exhibiting n-type thermoelectric properties," Small, vol. 13, p. $1603420,2017$.

[44] M. D. Clark, S. Subramanian, and R. Krishnamoorti, "Understanding surfactant aided aqueous dispersion of multi-walled carbon nanotubes," Journal of Colloid and Interface Science, vol. 354, pp. 144-151, 2011.

[45] G. Wu, Z.-G. Zhang, Y. Li, C. Gao, X. Wang, and G. Chen, "Exploring high-performance n-type thermoelectric composites using amino-substituted rylene dimides and carbon nanotubes," ACS Nano, vol. 11, pp. 5746-5752, 2017.

[46] Y. Nonoguchi, Y. Iihara, K. Ohashi, T. Murayama, and T. Kawai, "Air-tolerant fabrication and enhanced thermoelectric performance of n-type single-walled carbon nanotubes encapsulating 1,1' -bis(diphenylphosphino) ferrocene," Chemistry, an Asian Journal, vol. 11, pp. 2423-2427, 2016.

[47] T. Fujigaya, "Development of thermoelectric conversion materials using carbon nanotube sheets," Bulletin of the Chemical Society of Japan, vol. 92, pp. 400-408, 2019.

[48] T. Fukumaru, T. Fujigaya, and N. Nakashima, "Development of n-type cobaltocene-encapsulated carbon nanotubes with remarkable thermoelectric property," Scientific Reports, vol. 5, p. 7951, 2015.

[49] Y. Liu, Q. Dai, Y. Zhou et al., "High-performance n-type carbon nanotube composites: improved power factor by optimizing the acridine scaffold and tailoring the side chains," ACS Applied Materials \& Interfaces, vol. 11, pp. 29320-29329, 2019.

[50] C. K. Mytafides, L. Tzounis, G. Karalis, P. Formanek, and A. S. Paipetis, "High-power all-carbon fully printed and wearable SWCNT-based organic thermoelectric generator," ACS Applied Materials \& Interfaces, vol. 13, pp. 11151-11165, 2021.

[51] M. Wong, M. Paramsothy, X. J. Xu, Y. Ren, S. Li, and K. Liao, "Physical interactions at carbon nanotube-polymer interface," Polymer, vol. 44, pp. 7757-7764, 2003.
[52] Y.-S. Li, J.-L. Liao, S.-Y. Wang, and W.-H. Chiang, "Intercalation-assisted longitudinal unzipping of carbon nanotubes for green and scalable synthesis of graphene nanoribbons," Scientific Reports, vol. 6, p. 22755, 2016.

[53] R. M. F. Fernandes, B. Abreu, B. Claro et al., "Dispersing carbon nanotubes with ionic surfactants under controlled conditions: comparisons and insight," Langmuir, vol. 31, pp. 10955-10965, 2015.

[54] B. White, S. Banerjee, S. O'Brien, N. J. Turro, and I. P. Herman, "Zeta-potential measurements of surfactant-wrapped individual single-walled carbon nanotubes," Journal of Physical Chemistry C, vol. 111, pp. 13684-13690, 2007.

[55] J. W. Jo, J. W. Jung, J. U. Lee, and W. H. Jo, "Fabrication of highly conductive and transparent thin films from singlewalled carbon nanotubes using a new non-ionic surfactant via spin coating," ACS Nano, vol. 4, pp. 5382-5388, 2010.

[56] V. A. Sinani, M. K. Gheith, A. A. Yaroslavov et al., "Aqueous dispersions of single-wall and multiwall carbon nanotubes with designed amphiphilic polycations," Journal of the American Chemical Society, vol. 127, pp. 3463-3472, 2005.

[57] V. T. Tiong, N. D. Pham, T. Wang et al., "Octadecylaminefunctionalized single-walled carbon nanotubes for facilitating the formation of a monolithic perovskite layer and stable solar cells," Advanced Functional Materials, vol. 28, p. 1705545, 2018.

[58] M. Baibarac, I. Baltog, C. Godon, S. Lefrant, and O. Chauvet, "Covalent functionalization of single-walled carbon nanotubes by aniline electrochemical polymerization," Carbon, vol. 42, pp. 3143-3152, 2004.

[59] B. A. Jones, M. J. Ahrens, M. H. Yoon, A. Facchetti, T. J. Marks, and M. R. Wasielewski, "High-mobility air-stable n-type semiconductors with processing versatility: dicyanoperylene3,4:9,10-bis (dicarboximides)," Angewandte Chemie, vol. 116, pp. 6523-6526, 2004.

[60] A. Giovannitti, C. B. Nielsen, D.-T. Sbircea et al., "N-type organic electrochemical transistors with stability in water," Nature Communications, vol. 7, p. 13955, 2016.

[61] C. J. An, Y. H. Kang, H. Song, Y. Jeong, and S. Y. Cho, "Highperformance flexible thermoelectric generator by control of electronic structure of directly spun carbon nanotube webs with various molecular dopants," Journal of Materials Chemistry A, vol. 5, pp. 15631-15639, 2017.

[62] X. Mao, Z. Li, Y. Liu et al., "Tuning the structure of boranenitrogen derivatives towards high-performance carbon nanotubes-based n-type thermoelectric materials," Chemical Engineering Journal, vol. 405, article 126616, 2021.

[63] Y. Zou, P. Tan, B. Shi et al., "A bionic stretchable nanogenerator for underwater sensing and energy harvesting," Nature Communications, vol. 10, p. 2695, 2019. 